\title{
A heuristic for rationing inventory in two demand classes with backlog costs and a service constraint
}

Daqin Wang, Ou Tang and Jiazhen Huo

\author{
Linköping University Post Print
}

Tweet

N.B.: When citing this work, cite the original article.

Original Publication:

Daqin Wang, Ou Tang and Jiazhen Huo, A heuristic for rationing inventory in two demand classes with backlog costs and a service constraint, 2013, Computers \& Operations Research, (40), 12, 2826-2835.

http://dx.doi.org/10.1016/j.cor.2013.06.001

Copyright: Elsevier

http://www.elsevier.com/

Postprint available at: Linköping University Electronic Press

http://urn.kb.se/resolve?urn=urn:nbn:se:liu:diva-100967 


\title{
A heuristic for rationing inventory in two demand classes with backlog costs and a service constraint
}

\author{
Daqin Wang ${ }^{\mathrm{a}, \mathrm{b}, *} \quad$ Ou Tang ${ }^{\mathrm{b}, \mathrm{a}} \quad$ Jiazhen Huo $^{\mathrm{a}}$ \\ ${ }^{\mathrm{a}}$ The school of Economics and Management, Tongji University, Shanghai 200092, China \\ ${ }^{\mathrm{b}}$ Division of Production Economics, Department of Management and Engineering, Linköping \\ University, Linköping, SE-581 83, Sweden
}

\begin{abstract}
We study the rationing policy in an inventory system with two demand classes and different service criteria for backorders. Due to the difference of customer values, system performance sometimes has to be measured with a mixture of penalty cost and service level in managing inventory. With a continuous review $(r, Q)$ system, we develop a critical level rationing policy in which a threshold mechanism is adopted to allocate backorders when multiple outstanding orders exist. Due to the problem complexity, a heuristic is developed based on the principle that both demand classes are served with respective target service levels. We also introduce bounds so that the search ranges of decision variables become restrictive. The numerical examples indicate an excellent performance of our heuristic. In addition, when ordering cost is medium or high, the threshold clearing mechanism has the same results as the optimal one. When ordering cost is small (set to zero), different clearing mechanisms should be used depending on the priorities of demand classes. Further analysis indicates that transforming the service constraint into a cost parameter and then applying the existing algorithm will not be a good approach for this problem with mixed performance criteria. It either increases the costs or violates the service constraint. This study also shows the importance of applying rationing policy when high priority class has a low demand volume, target service levels between two classes have a large gap, or replenishment lead time is long. The results of this study should enhance our understanding of how to implement rationing policies in practice.
\end{abstract}

(Keywords: multiple demand classes, rationing policy, mixed service criteria, service constraint)

\footnotetext{
* Corresponding author. Tel.: +46 13 282355; fax: +46 13 281101. Email address: daqin.wang@liu.se.
} 


\section{Introduction}

In the traditional inventory models, demand is often considered to be homogenous. But in practice, demand may come from different customer groups, each of which has its own properties such as demand rate, requirement for service level, response to stockouts and value preference. If the traditional inventory control models are applied and customers are treated without considering their individual requirements, there should be a mismatch between demand and supply. For instance, customers from high priority class receive low service levels, or vice versa. This inspires the research on inventory control with multiple demand classes. Since Veinott (1965) first introduced a rationing policy to distinguish service levels for different demand classes, there is a growing body of literature in this area.

Along with the development of service industry in recent years, operations management becomes even more customer-orientated. Nowadays, customers not only have various values regarding the same products, but also have different measures for evaluating service performance. For example, in the retailing industry, vendors usually supply several retailers of different scales. In the Chinese market, vendors are often required to guarantee a minimal service level during the contract period in order to contract with large retailers such as Bailian and Wal-Mart. On the other hand when vendors deal with small retailers such as local convenience stores, they are likely facing a "lost business" or "compensate for the delayed delivery" case when stockouts occur. Or alternatively, vendors can claim penalty costs for lost sales or backlogs. The second example is found in two steel companies, one in China and one in Sweden. Both companies have two to five important customers accounting for a large proportion of total demand. They are often required to fulfil a predefined service level to those VIPs. When the production capacity is not enough, they will reject orders from ordinary customers and lose the potential profits. The third example is a large Swedish company producing hardware for mobile networks. The spare parts can be either sold as normal goods in the market, or supplied to customers due to the warranty issue. In the former, stockouts reduce the economic income whereas in the latter, stockouts are limited (in terms of time and/or quantity) based on the contract. In all the above illustrated industrial examples, there are usually mixed service criteria, one associated with cost and the other with service constraint, when we are dealing with inventory problems having multiple demand classes.

Although many researches have focused on the problem of multiple demand classes, less attention has been paid to the mixed service criteria. Existing literature often deal with demand classes with the same measures for controlling backorders, i.e. the performance measure is based on either penalty costs or service constraints. Even though we can transform the service level to a penalty cost and apply the existing cost-related methods (for instance Deshpande et al., 2003) to solve the mixed criteria problem, whether this approach is appropriate still needs to be investigated.

The above background provides the motivation of the current study. In this paper, we study inventory control policies with two demand classes. More specifically, a penalty cost is paid to one class when backorder occurs, whereas a minimal service level is promised to the other. We aim at studying the interaction between service levels and costs in such a system so as to obtain insights for management. We also focus on how to obtain the optimal rationing decisions in such a mixed criteria system. Furthermore, we examine in which circumstances the rationing policy should bring advantages to inventory management.

The structure of this paper is organised as follows. After this introductory section, we review 
the relevant literature in order to clarify the research gap in Section 2. In Section 3, we develop the rationing model. Due to the complexity of the problem (multiple outstanding orders and complicated backorders clearing mechanism), we adopt the threshold mechanism (Deshpande et al., 2003) to formulate the expressions of inventory and service levels. Bounds are developed to expedite the search procedure in our heuristic. Furthermore, numerical analysis is given in Section 4 to examine the performance of the heuristic. Finally, we draw our conclusions and present the ideas for future study in Section 5.

\section{A brief literature review}

Inventory control with multiple demand classes has been studied for decades since Veinott (1965), with the aim to distinguish customer values in individual groups and thereafter improve economic consequences in business systems. A large body of literature has studied this problem in different settings. Our paper studies multiple backorder classes extended from a continuous $(r, Q)$ system, which will be one focus of literature review. In addition, we extend the modelling approach and solution method for different performance measures such as cost and service constraint. The representative models will be included in this brief literature review.

Current research has a strong connection with the research stream on rationing policies using continuous review $(r, Q)$ system. Nahmias and Demmy (1981) first study the rationing policy in a continuous review environment. They develop the expressions for backorders and service levels. However the optimal policy has not been discussed. In addition, they introduce the assumption of having at most one outstanding order. This assumption avoids the replenishment allocation problem, i.e. the need for a clearing mechanism for backorders of difference classes, thus the analysis is simplified. Moon and Kang (1998) extend the research to compound Poisson distributed demand. Kleijn and Dekker (1998) review the studies on inventory models with multiple demand classes. They indicate that one difficulty in deriving optimal solution in a continuous review system is due to the possible existence of multiple outstanding orders. In this case, inventory position does not have a straightforward relation with inventory level, but depends on the clearing mechanism for different classes. Deshpande et al. (2003) make a breakthrough in dealing with this problem. They design a threshold clearing mechanism, which brings the advantage of obtaining closed-form expressions to performance measures. Further analysis shows that such a threshold mechanism can be used as a good approximation for the priority clearing mechanism, which is often considered as the optimal policy. The threshold method will be extended to deal with our problem in this study.

One difference between our research and existing ones is due to our consideration of specific service requirements of different classes. The purpose of rationing inventory for multiple demand classes is to distinguish customers with an aim of improving system performance. But existing studies pay less attention to the unique characteristics of different customers. Although many papers consider multiple demand classes, they still apply the same performance measure. For example, there are papers assigning various backorder penalty costs to different demand classes (Dekker et al., 1998, Melchiors et al., 2000, and Deshpande et al., 2003). Other papers such as Cohen et al. (1988) and Duran et al. (2008) have a focus on different service levels. There is one paper focusing on two service criteria (Chew et al, 2009). They use simulation to evaluate a $(\underline{r}, Q)$ system with $m$ customer classes. The key performance indexes are total cost and service levels. The service is defined as the backorder number of each class. However we employ fill-rate as a service constraint for one class. Other studies on inventory rationing include periodic review systems (Topkis, 1968), lost sales (Melchiors et 
al., 2000), dynamic rationing (Melchiors, 2003, Teunter and Haneveld, 2008, Fadiloglu and Bulut, 2010) and multi-echelon (Axsäter et al., 2004, Paul and Rajendran, 2011). For a recent review on inventory rationing policies with various extensions, we refer to Teunter and Haneveld (2008).

In summary we have observed in practice that customers may have different demand characteristics and performance preferences, as mentioned in the introduction. But in the inventory rationing literature, less attention has been paid to the situation of having mixed service criteria, specifically there is no study investigating the case with a mixture of service requirements and penalty costs. How can we obtain the optimal solutions, and whether previous solution procedures can still be readily modified to such a case? These open research questions are worth to be tackled and our current study hopefully will fill a research gap in the existing literature.

\section{Rationing model}

\subsection{System description}

In this study, we consider a continuous review $(r, Q)$ system with two demand classes, each of which follows a Poisson demand process with rate $\lambda_{i}(i=1,2)$. Replenishment lead time is a constant, during which any unmet demand is backlogged. One important characteristic of this model is that a minimal service level $w_{1}$ is required by Class 1 customers, whereas a penalty cost $\pi_{2}$ (per unit per time unit) will be charged for a backlogged demand of Class 2 . For the purpose of simplicity, we focus on the rationing policy with a static critical level $c$ : once on-hand inventory falls to $c$, demand from low priority class begins to be backlogged. Our objective is then to solve the problem in the extended continuous review $(Q, r, c)$ system in order to minimise the expected cost with the restriction of service constraint.

Note that the Poisson is not the only distribution for illustrating stochastic demand process. The main limitation is its single parameter, which further makes its variance equal to mean. In some cases, for instance the contracted retailers like Bailian and Wal-Mart mentioned in previous section, demand may have a relatively small variance and differentiating variance and mean will be more appropriate. Nevertheless, the closed-form expressions for inventory, stockout and service level will then be difficult to derive, and further analysis will be more complicated and less transparent in our problem settings. Therefore we keep the assumption of Poisson distribution, as in many studies in early literature. This also brings the convenience to compare our model with some benchmark approaches.

The following notations are used:

$i$ : index for classes, $i=1,2$;

$\lambda_{i}$ : demand rate for Class $i$, and the total rate is $\lambda=\lambda_{1}+\lambda_{2}$;

$w_{1}$ : minimal service level (fill-rate) requirement for Class 1 ;

$\pi_{2}$ : penalty cost per unit per unit time for Class 2 ;

$F_{i}:$ actual or expected service level (fill-rate) for Class $i$;

$L:$ replenishment lead time;

$K$ : ordering cost; 
$h$ : holding cost per unit per unit time;

$H$ : average holding cost in the system;

$u_{i}$ : average backorders for Class $i$ in the system;

$A C:$ average cost in the system;

$Q$ : ordering quantity;

$r$ : reorder point;

$c$ : critical rationing level.

Also we define $B I\left(\alpha_{i} ; n ; n_{i}\right)=C_{n}^{n_{i}} \alpha_{i}^{n_{i}}\left(1-\alpha_{i}\right)^{n-n_{i}}$. as the probability that we have exactly $n_{i}$ class $i$ demands from a demand stream of $n$ customers. Define $p(x, \mu)$ as the probability mass function and $P(x, \mu)=\sum_{i=0}^{x} p(i, \mu)$ as the cumulative distribution function of Poisson distribution.

We can first formulate a general model for our problem as

$$
\begin{gathered}
\text { Minimize } A C(Q, r, c)=K \frac{\lambda}{Q}+H(Q, r, c)+\pi_{2} u_{2}(Q, r, c) \\
\text { s.t. } F_{1}(Q, r, c) \geq w_{1}
\end{gathered}
$$

The aim is to obtain the optimal $(Q, r, c)$ to minimise the average cost consisting of ordering cost, holding cost and penalty cost of Class 2 , with a service constraint required by Class 1 .

One immediate problem is that the priority of two classes is not pre-defined, because we are dealing with mixed service criteria. It is well known that in the classic $E O Q$ model with deterministic demand and backorders, the penalty cost (such as $\pi_{2}$ ) can be transformed into a service level $\pi_{2} /\left(h+\pi_{2}\right)$ which actually is the target service level in the optimal system (cf. Zipkin, 2000, pp47). With this principle, we propose to compare the values of $w_{1}$ and $\pi_{2} /\left(h+\pi_{2}\right)$ for defining the demand priority. This finally leads to two cases: $w_{1}>\pi_{2} /\left(h+\pi_{2}\right)$ and $w_{1} \leq \pi_{2} /\left(h+\pi_{2}\right)$.

In the following we will present the model development for the case $w_{1}>\pi_{2} /\left(h+\pi_{2}\right)$, i.e. Class 1 has a high priority, because important customers are often guaranteed a service level in practice (cf. our examples in Section 1). Note that the model development of the case $w_{1} \leq \pi_{2} /\left(h+\pi_{2}\right)$ can follow the same procedure, and the main results will be summarised in Section 3.4.

\subsection{Model formulation with the threshold clearing mechanism}

As mentioned before, when dealing with the stochastic $(Q, r, c)$ system, one difficulty is: several outstanding orders may exist simultaneously, for instance when replenishment lead time is long. Alternatively, when ordering quantity $Q$ is small, the replenishment may not be enough to clear backorders of both classes. This creates an allocation problem which complicates the analysis. It has been stated in literature that in such a $(Q, r, c)$ policy, the optimal clearing mechanism is to clear first the backorders of high priority customers. However, this priority clearing scheme is intractable due to a lack of closed-form expressions for the inventory level. Since we also apply the threshold method in this study, we briefly 
repeat in this section the definition and some relevant results developed by Deshpande et al. (2003). This should bring convenience for further model development and result discussion. For more details and thorough discussions on this approach, please refer to the original work by Deshpande et al. (2003).

The threshold clearing mechanism is defined as: the replenishment is used to clear backorders according to their occurrence sequence as if $Q$ already exists a lead time ago. Figure 1 illustrates the typical events during a cycle where Class 1 has higher priority than Class 2 . The $j$ th replenishment order is placed at time $t_{j}$, causing the inventory position to rise to $r+Q$. $t_{u_{i}}$ indicates the time when demand starts to be backlogged for Class $i . t_{c_{j}}$ is defined as the time when $(r+Q-c)$ th demand happens in the interval $\left(t_{j}, t_{j}+L\right)$. During the interval $\left(t_{j}, t_{c_{j}}\right)$, the demand is assumed to be fulfilled by first-come-first-serve principle so that the backorders are cleared by the replenishment according to the arrival sequences. Then the remaining replenishment is used to fulfil the backorders of Class 1 occurred during the interval $\left(t_{c_{j}}, t_{j}+L\right)$. The backorders of Class 2 during this interval are carried until next replenishment arrives. The main difference between the priority and threshold clearing mechanisms is due to the interval $\left(t_{j}, t_{c_{j}}\right)$, during which the priority mechanism always clears backorders of high priority class first, while the threshold mechanism clears backorders according to the arrival sequence. The threshold mechanism should lead to more backorders of high priority class. Even though it is not an optimal policy, the threshold mechanism brings the possibility of formulating expressions of the inventory level.

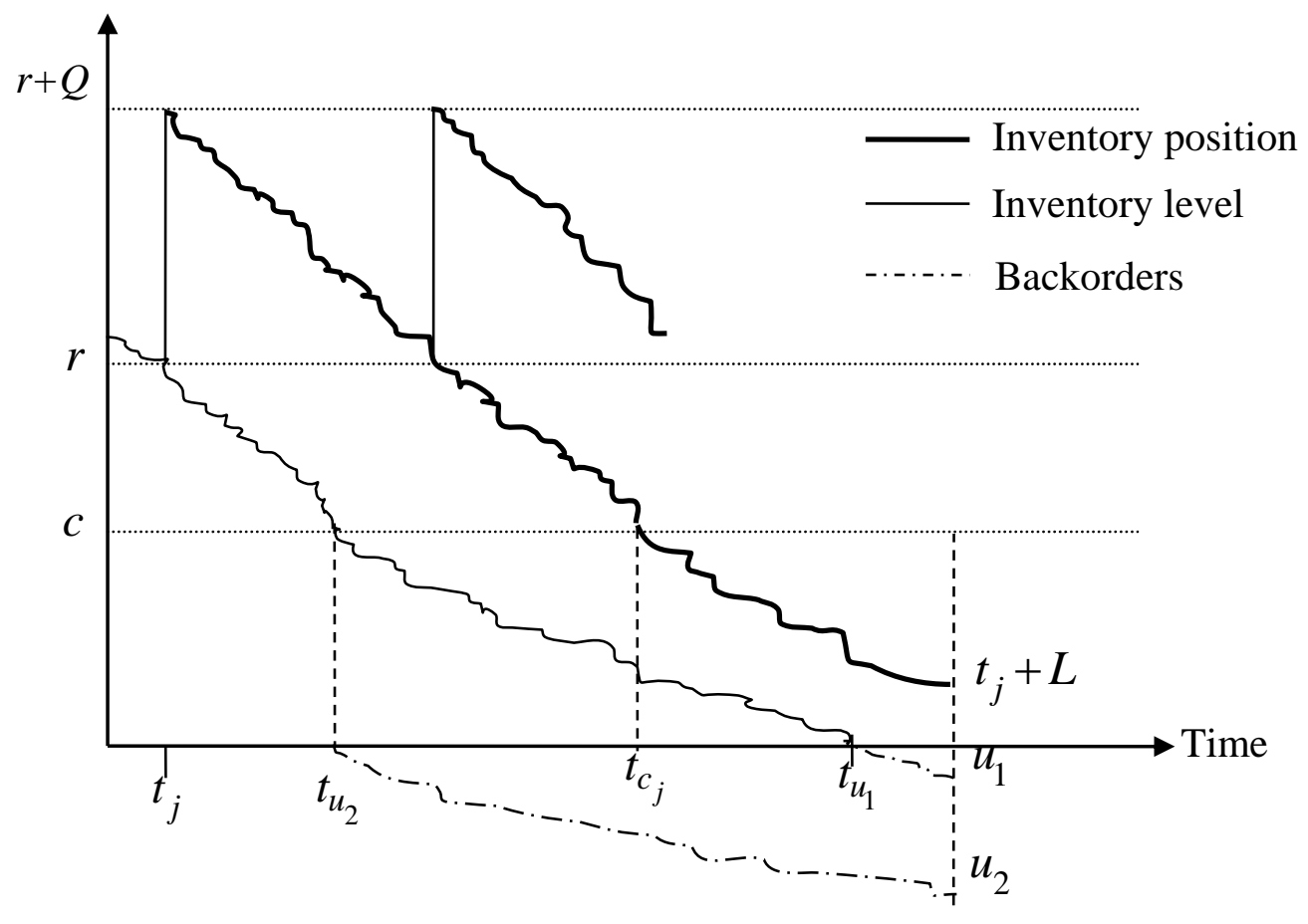

Figure 1. The threshold clearing mechanism (Adapted from Deshpande et al., 2003).

In the system described above, the specific reorder time $t_{j}$ can be generalised to any random time $t$. Since the formulae are adopted from Deshpande et al. (2003) with some 
modifications, we omit the derivations and only present the major results.

First, the expected inventory holding cost can be formulated as

$$
\begin{aligned}
H(Q, r, c) & =h \sum_{y=r+1}^{r+Q} E\left\{y-D(t, t+L)+\left[D_{1}\left(t_{c}, t+L\right)-c\right]^{+}+D_{2}\left(t_{c}, t+L\right)\right\} \\
& =h\left\{\frac{Q+1}{2}+r-\lambda L+u_{1}(Q, r, c)+u_{2}(Q, r, c)\right\}
\end{aligned}
$$

The expected backorders can be defined as

$$
u_{i}(Q, r, c)=\frac{1}{Q} \sum_{y=r+1}^{r+Q} b_{i}(y, c)
$$

where

$$
\begin{array}{r}
b_{1}(y, c)=\sum_{x=y}^{\infty} p(x ; \lambda L)\left[\sum_{j=0}^{x-y} j B I\left(\alpha_{1} ; x-(y-c) ; c+j\right)\right] \\
b_{2}(y, c)=\left\{\begin{array}{rc}
\sum_{x=(y-c)}^{\infty} \alpha_{2}(x-(y-c)) p(x ; \lambda L), & c \leq y \\
\lambda_{2} L+\alpha_{2}(c-y), & c>y
\end{array}\right.
\end{array}
$$

Since the service constraint needs to be considered in our model, the expected service level should be developed as

$$
F_{i}(Q, r, c)=\frac{1}{Q} \sum_{y=r+1}^{r+Q} f_{i}(y, c)
$$

where

$$
\begin{array}{r}
f_{1}(y, c)=1-\sum_{x=y}^{\infty} \sum_{j=0}^{x-y} B I\left(\alpha_{1} ; x-(y-c) ; c+j\right) p(x ; \lambda L) \\
f_{2}(y, c)= \begin{cases}P(y-c-1 ; \lambda L), & c \leq y \\
0, & c>y\end{cases}
\end{array}
$$

Thus in our problem, we further define $G(y, c)=h(y-\lambda L)+h b_{1}(y, c)+\left(h+\pi_{2}\right) b_{2}(y, c)$, and rewrite the average cost function as

$$
A C(Q, r, c)=\frac{K \lambda+\sum_{y=r+1}^{r+Q} G(y, c)}{Q}
$$

Notice that there is no penalty cost of Class 1 in $G(y, c)$. The above expression presents a similar structure as the one in the classic $(Q, r)$ model, but with an additional service constraint (Equation 2). Furthermore, the expressions above are valid when Class 1 has higher priority than Class 2. In the case when Class 2 has a high priority, we should have similar expressions which are omitted from the paper.

\subsection{Discussion on optimisation}

The traditional method to solve an optimisation problem indicated as Equations 1 and 2 is Karush-Kuhn-Tucker (KKT) approach. However, the decision variables in the inventory model are discrete (integers) and the convexity of the objective function is indefinite. In fact in the literature of similar inventory models, search method is often used to find the "optimal" decision variables such as $(Q, r, c)$. In the study of Deshpande et al. (2003) which considers 
penalty costs for both demand classes, they prove the convexity of $G(y, c)$ in $y$ thus the search algorithm in the classic $(Q, r)$ model can be used. In fact the method of Federgruen and Zheng (1992) is used to obtain the optimal ordering quantity $Q$ and reorder point $r$ with any given $c$. The optimal $c$ is then obtained by performing a complete search on all possible values (basically between 0 and $Q+r$ when the demand process is Poisson). In our problem one penalty cost is replaced by a service constraint. One intuitively straightforward method will be: transform $w_{1}$ to a penalty cost $\pi_{1}=h w_{1} /\left(1-w_{1}\right)$ for Class 1 and then use the method of Deshpande et al. (2003) to solve the problem. However, the structure of the model is changed due to mixed service criteria, and the optimal solutions obtained by Deshpande et al. (2003) are no longer guaranteed to be optimal in our model. When the service requirement becomes a hard constraint, the feasible solution space will be restricted. Thus the method based on the modified penalty costs may not always lead to a satisfied service level. Some results will be presented in the numerical examples to support our arguments.

In fact since $G(y, c)$ is convex in $y$ for any $c$, we can refer to our mixed criteria model as a $(Q, r)$ model with a service constraint. However, there exists no simple method to calculate optimal decision variables in a classic $(Q, r)$ system with a service constraint. Platt et al. (1997) provide a tractable $(Q, r)$ heuristic for service constrained problem. But their method should follow the assumption that the demand during lead time is normally distributed. Agrawal and Seshadri (2000) propose a method for arbitrary demand distribution during lead time and derive bounds for searching the optimal $(Q, r)$. Their study has an implicated assumption that the demand distribution is continuous, which cannot be applied to our model. Also note that in our model the rationing level $c$ influences the demand distribution during the lead time and it complicates the problem immediately. Thus the above mentioned methods (or their principle) cannot be used in our case. In short, we need to develop a new algorithm for solving the problem.

\subsection{Heuristic}

In this section, we present our heuristic approach which aims to obtain near optimal solutions in the $(Q, r, c)$ system. The heuristic is based on a search method: with a sequence of $c, r$ and $Q$. Note that this search sequence is different compared with the one in Deshpande et al. (2003). Based on the specific problem structure in our model, we can develop bounds or stop conditions in the search method for the decision variables, and thus the search efficiency can be significantly improved.

The heuristic is inspired as follows. We know that in the classic EOQ model with penalty cost $\pi$ for backorders, the optimal solutions always provide the target service level $\pi /(h+\pi)$ (cf. Zipkin, 2000, pp47). Moreover, even in $(r, Q)$ model with discrete stochastic unit demand, we have the fill-rate $F$ with optimal $r$ as $F\left(r^{*}\right) \leq \pi /(h+\pi)<F\left(r^{*}+1\right)$, which implies the optimal solutions intend to provide service close to $\pi /(h+\pi)$ (cf. Axsäter, 2006, pp103). We utilise this property to select decision variables so that the service levels of two classes are approaching their respective target values. The main procedure of the heuristic is: with a given $Q$, we develop the bounds of $r$ and $c$ and search for their values in order to make service level $F_{i}$ close to the respective service levels $w_{1}$ and $\pi_{2} /\left(h+\pi_{2}\right)$. Since 
demand is discrete and the decision variables are integers, the target service levels probably cannot be reached exactly. More specifically, $F_{1}$ can only approach as the nearest value no less than the target value due to the inequality service constraint, while $F_{2}$ can approach from either side to its target value. Finally $Q$ is searched. Lemmas 1 and 2 are needed for developing our heuristic.

Lemma 1. The service level of the high priority class $F_{h i g h}(Q, r, c)$ is increasing in $r$ and increasing in $c$, and the service level of the low priority class $F_{\text {low }}(Q, r, c)$ is increasing in $r$ and decreasing in $c$.

In the above Lemma, $F_{\text {high }}$ and $F_{\text {low }}$ indicate the service levels of high and low priority classes, respectively. This lemma has been proved in Deshpande et al. (2003).

We also need to develop the next lemma for our heuristic.

Lemma 2. $A C(Q, r, c)$ is convex in $c$ for fixed $Q$ and $r$.

Proof. $b_{i}(y, c)$ is convex in $c$ for a fixed $y$. Based on Equation 10 and $G(y, c)=h(y-\lambda L)+h b_{1}(y, c)+\left(h+\pi_{2}\right) b_{2}(y, c)$, we can conclude that $A C(Q, r, c)$ is convex in $c$ for a fixed $y$. In addition, $y$ is a function of $Q$ and $r$.

Now, we further develop the bounds as follows.

Case 1, $w_{1}>\pi_{2} /\left(h+\pi_{2}\right)$.

In this case, Class 1 has a high priority. As mentioned before, with a given $Q$, we aim at finding $r$ and $c$, in order to make $F_{1}(Q, r, c)$ and $F_{2}(Q, r, c)$ as close as possible to $w_{1}$ and $\pi_{2} /\left(h+\pi_{2}\right)$, respectively. Figure 2 presents the curves of $F_{i}(Q, r, c)$ with different values of $r$ and $c$.

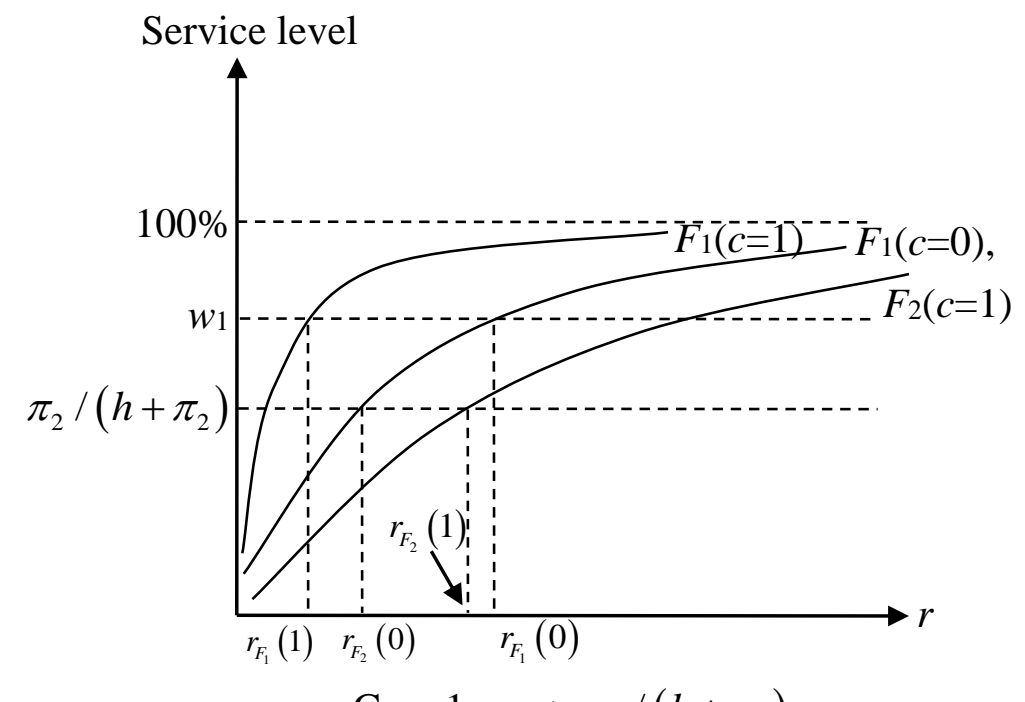

Case 1. $w_{1}>\pi_{2} /\left(h+\pi_{2}\right)$

Figure 2. The curves of $F_{i}$ in Case 1.

Now we have the following lemma. 
Lemma 3. When $w_{1}>\pi_{2} /\left(h+\pi_{2}\right)$, with a given $Q$, the lower bound of $r$ is $r^{l}(Q)=\max \left\{r \mid \sum_{y=r+1}^{r+Q} P(y-1, \lambda L) / Q \leq \pi_{2} /\left(h+\pi_{2}\right)\right\}$, and the upper bound of $r$ is $r^{u}(Q)=\min \left\{r \mid \sum_{y=r+1}^{r+Q} P(y-1, \lambda L) / Q \geq w_{1}\right\}$.

Proof. According to the principle described above, we aim to make $F_{i}$ approach to its target service level. We first focus on letting $F_{1}$ approach to $w_{1}$ from above due to the service constraint. Since $F_{1}(Q, r, c)$ increases in $r$, then with any $c$ there exists a minimal $r$ satisfying the constraint $F_{1}(Q, r, c) \geq w_{1}$. We denote it as $r_{F_{1}}(c)=\min \left\{r \mid F_{1}(Q, r, c) \geq w_{1}\right\}$. As $F_{1}$ increases in $c$ (Lemma 1 ), we have $r_{F_{1}}(c)$ decreasing in $c$, which indicates $r_{F_{1}}(0)=\max \left\{r_{F_{1}}(c) \mid c \geq 0\right\}$. Since $F_{1}(Q, r, 0)=\sum_{y=r+1}^{r+Q} P(y-1, \lambda L) / Q$, we denote the upper bound of $r$ as $r^{u}(Q)=r_{F_{1}}(0)=\min \left\{r \mid \sum_{y=r+1}^{r+Q} P(y-1, \lambda L) / Q \geq w_{1}\right\}$.

The lower bound of $r$ is obtained in a similar way. We aim at making $F_{2}$ close to $\pi_{2} /\left(h+\pi_{2}\right)$ from below so as to guarantee the lower bound of $r$. Since $F_{2}$ increases in $r$, for any $c$, we have $r_{F_{2}}(c)=\max \left\{r \mid F_{2}(Q, r, c) \leq \pi_{2} /\left(h+\pi_{2}\right)\right\}$. As $F_{2}$ also decreases in $c$, we have $r_{F_{2}}(0)=\min \left\{r_{F_{2}}(c) \mid c \geq 0\right\}$. When $c=0, \quad F_{2}(Q, r, 0)=\sum_{y=r+1}^{r+Q} P(y-1, \lambda L) / Q$. Finally we can denote the lower bound of $r$ as $r^{l}(Q)=r_{F_{2}}(0)=\min \left\{r \mid \sum_{y=r+1}^{r+Q} P(y-1, \lambda L) / Q \leq \pi_{2} /\left(h+\pi_{2}\right)\right\}$.

Furthermore, since $\sum_{y=r+1}^{r+Q} P(y-1, \lambda L) / Q$ is increasing in $r$, it is easy to see $r^{l} \leq r^{u}$. Thus we have obtained the search range of $r$, within which $F_{i}$ is restricted to its target value. $\square$

Lemma 4. When $w_{1}>\pi_{2} /\left(h+\pi_{2}\right)$, with given $Q$ and $r$, the lower bound of $c$ is $c^{l}(Q, r)=\min \left\{c \mid F_{1}(Q, r, c) \geq w_{1}\right\}$. We increase $c$ until $A C(Q, r, c)$ begins to increase.

Proof. $c$ is assumed to be a non-negative integer. As $F_{1}$ is increasing in $c$, we first need to increase $c$ to satisfy $F_{1}(Q, r, 0) \geq w_{1}$. Thus we have the lower bound of $c$ as $c^{l}(Q, r)=\min \left\{c \mid F_{1}(Q, r, c) \geq w_{1}\right\}$. As $A C(Q, r, c)$ is convex in $c$ (Lemma 2), we can stop increasing $c$ when $A C(Q, r, c)$ begins to increase. $\square$

Next, we develop lower bound and stop signal for searching $Q$. As we can observe (see Figure 3), the average cost (with optimal $r$ and $c$ ) $A C^{*}(Q)$ is not a smooth curve with respect to $Q$. It exhibits a general tendency of decreasing and then increasing pattern. However, there exist many local optima. These sudden changes of $A C$ value are associated with the changes of the optimal values of $r$ or $c$, which are restricted to be integers. Here again, a search method is suggested for obtaining $Q$. 


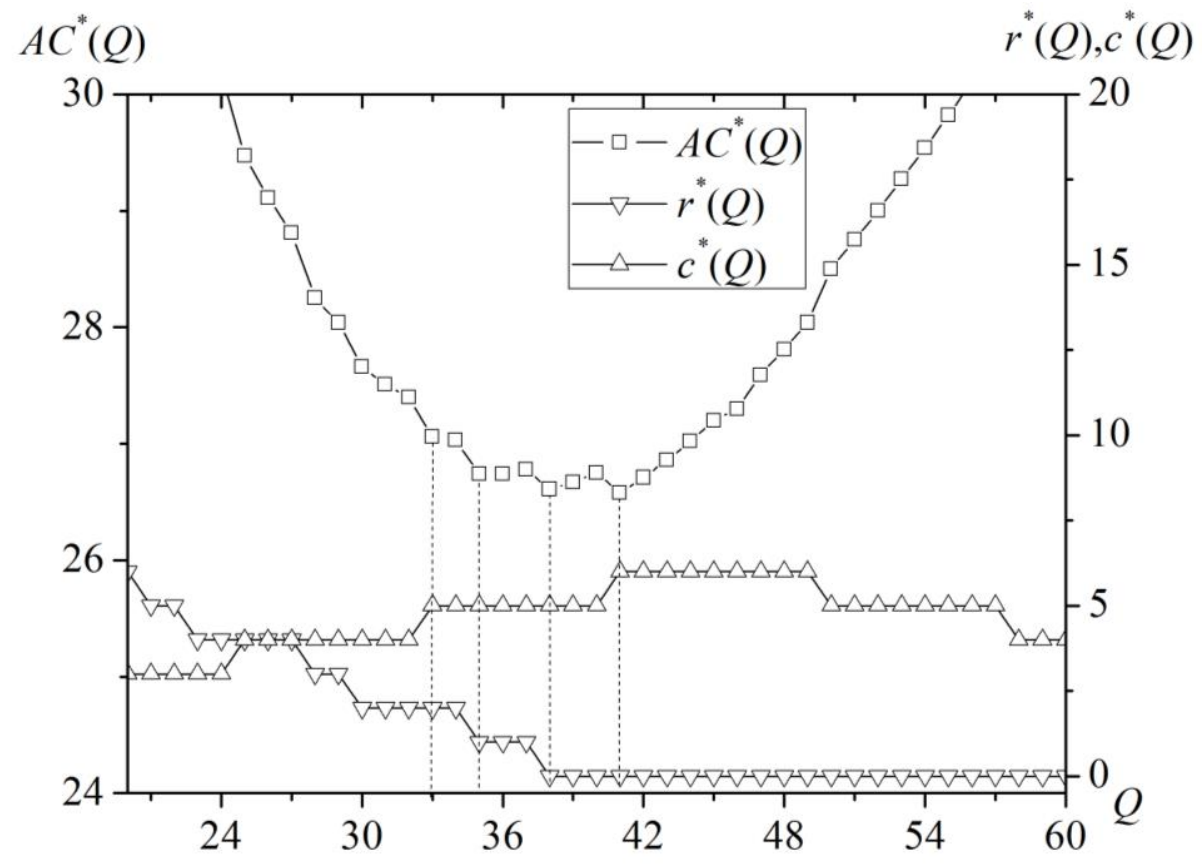

Figure 3. The curves of $A C^{*}(Q), r^{*}(Q)$ and $c^{*}(Q)$.

The lower bound for searching $Q$ should be $E O Q$. Even though we cannot provide a formal proof, the logic is illustrated below. We can divide the cost $A C$ (Equation 1) into two parts: the ordering cost $K \lambda / Q$, and the sum of holding cost and penalty cost $z_{1}=h[(Q+1) / 2+r-\lambda L]+h u_{1}+\left(h+\pi_{2}\right) u_{2}$. We draw the curves of these two parts in Figure 4. It is intuitively true that when $Q$ is getting large, the optimal reorder point $r$ and the backorders $u_{i}$ will decline and approach to zero. If we define $z_{2}=h[(Q+1) / 2-\lambda L]$, we can see that $z_{1}$ is approaching to $z_{2}$ as $Q$ increases, and in the meantime $z_{1}$ always has a smaller slope than $z_{2}$ (In Figure 4, the slope of $z_{1}$ is never more than 0.5). If we further ignore the constant term $h(1 / 2-\lambda L)$ in $z_{2}$, we obtain $z_{3}=h Q / 2$ which is the holding cost in the classic EOQ model. The sum of $K \lambda / Q$ and $z_{3}$ gets its minimal value when the slope of $K \lambda / Q$ equals the minus slope of $z_{3}$ at $E O Q=\sqrt{2 K \lambda / h}$. Since $z_{1}$ has a smaller slope than $z_{2}$, it also has a smaller slope than $z_{3}$. According to the necessary condition for optimisation, the sum of two functions achieves their minimal value when the sum of the slope values equals zero. Because $z_{1}$ has a smaller slope than $z_{3}$, the sum of $z_{1}$ and $K \lambda / Q$ must achieve the minimum on the right-side of EOQ. Thus EOQ should be the lower bound of $Q$. 


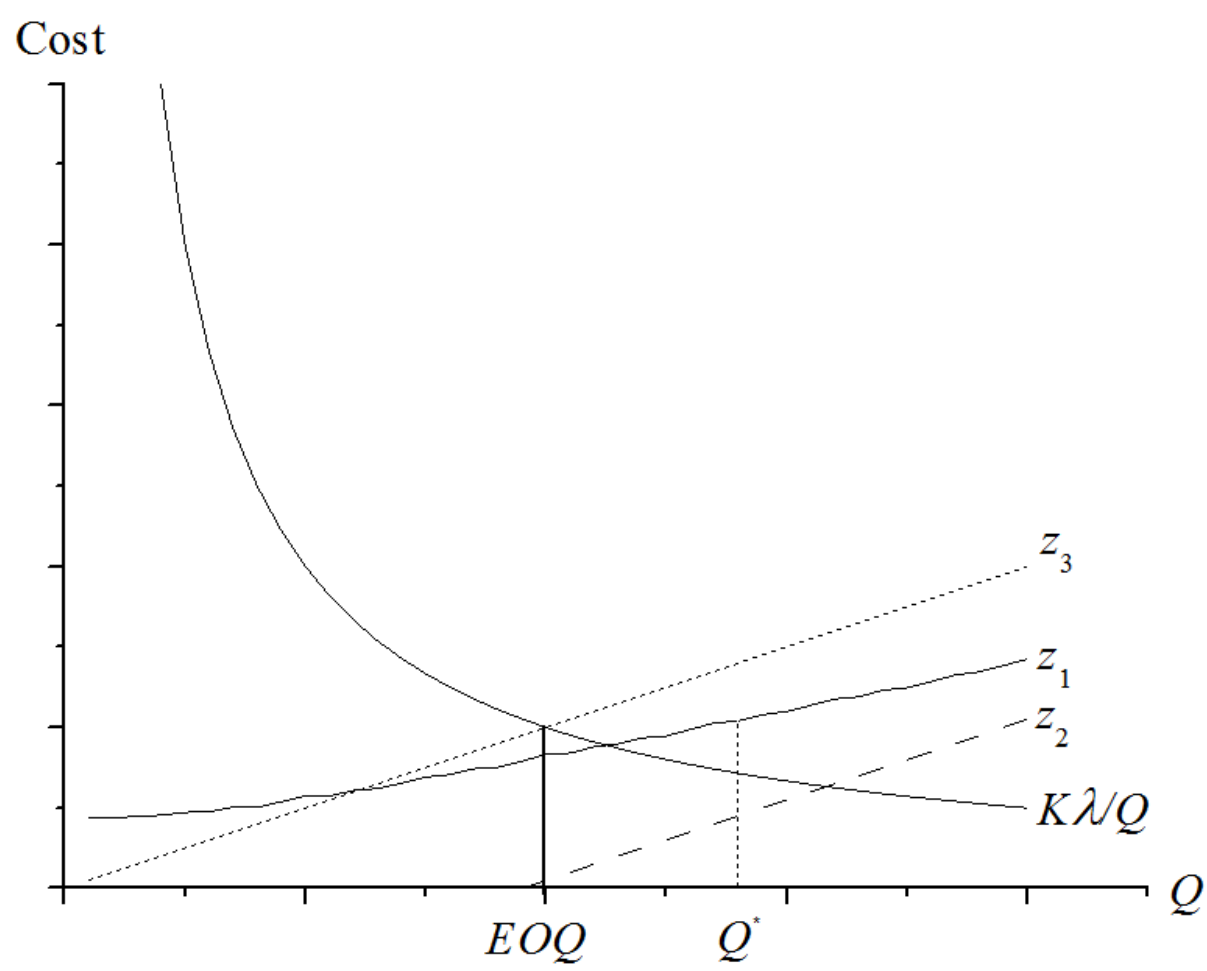

Figure 4. The lower bound of $Q$.

It is also hard to derive a closed-form expression for the upper bound of $Q$. However, we can develop a stop signal for searching $Q$ according to our observations. As mentioned before, $A C^{*}(Q)$ is not a smooth curve as $Q$ increases. In Figure 3, two lines denoted with triangle symbols are respectively the optimal $r$ and $c$ with a given $Q$ value. We can see that the changes of either $r$ or $c$ cause the "sudden" changes of the average cost, especially at the low part of the curve. According to our observation, $A C^{*}(Q)$ is initially decreasing monotonically when $Q$ increases from zero. When $Q$ increases further, $A C^{*}(Q)$ will increase, and then it may follow a value drop which leads to a local optimal cost. This "increase and drop" phenomenon then repeats. Furthermore, when $Q$ increases to a large value, the small "drops" do not necessarily decrease the $A C^{*}(Q)$, but rather slow down the increment speed of $A C^{*}(Q)$. This is due to the fact that, when $Q$ is sufficiently large, both optimal $r$ and $c$ are tending towards zero, $A C^{*}(Q)$ is predominately influenced by $Q$, and has less impacts from $r$ and $c$. Alternatively, we interpret it as: the cycle length is enlarged with a large $Q$. Although reducing the optimal $r$ leads to more penalty costs during a cycle, the average penalty cost will still decrease due to fewer cycles in a certain time horizon. Also a large $Q$ already provides both classes with a good service level during a cycle, further distinguishing of two demand classes may create only minor effects and thus a positive critical level may not be necessary anymore. Thus, with a large $Q$ value, $A C^{*}(Q)$ becomes monotonically increasing. Based on the above principle and observation, we suggest the following stop rules in searching $Q$.

We increase $Q$ unit by unit starting from the $E O Q$ value, and update the existing minimal value $A C^{*}$. Meanwhile, if we notice the change of either $r^{*}(Q)$ or $c^{*}(Q)$ values as $Q$ increasing, we compare the associated $A C^{*}(Q)$ value with the existing minimal one. If 
$A C^{*}(Q)$ is reduced, we update the new minimal value and continue searching. If $A C^{*}(Q)$ is bigger than the existing minimum, we stop our search and set the recorded minimal cost as the optimal one. In our later numerical examples, this rule always assures us to obtain the optimal $Q$.

Case 2, $w_{1} \leq \pi_{2} /\left(h+\pi_{2}\right)$.

In this case, Class 2 has a high priority. We have derived bounds for decision variables using the similar approach as in Case 1. We summarise here Lemmas 5 and 6 for calculating optimal $r$ and $c$. The optimal $Q$ is searched with the same principle.

Lemma 5. When $w_{1} \leq \pi_{2} /\left(h+\pi_{2}\right)$, with a given $Q$, the lower bound of $r$ is $r^{l}(Q)=\min \left\{r \mid \sum_{y=r+1}^{r+Q} P(y-1, \lambda L) / Q \geq w_{1}\right\}$, and the upper bound of $r$ is $r^{u}(Q)=\min \left\{r \mid \sum_{y=r+1}^{r+Q} P(y-1, \lambda L) / Q \geq \pi_{2} /\left(h+\pi_{2}\right)\right\}$.

Lemma 6. When $w_{1} \leq \pi_{2} /\left(h+\pi_{2}\right)$, with a given $r$, the lower bound of $c$ is $c^{l}=0$. We increase $c$ until $A C(Q, r, c)$ begins to increase or $F_{1}(Q, r, c) \leq w_{1}$.

Furthermore, we would emphasise that in Lemmas 3 and 5, the bounds of $r$ are independent of $c$. When $Q$ is given, the upper and lower bounds of $r$ become constant. This property brings the advantage in our search algorithm. Now, based on above discussion and lemmas, the heuristic algorithm for searching optimal $c, r$ and $Q$ is finally presented.

\section{Algorithm: Threshold heuristic}

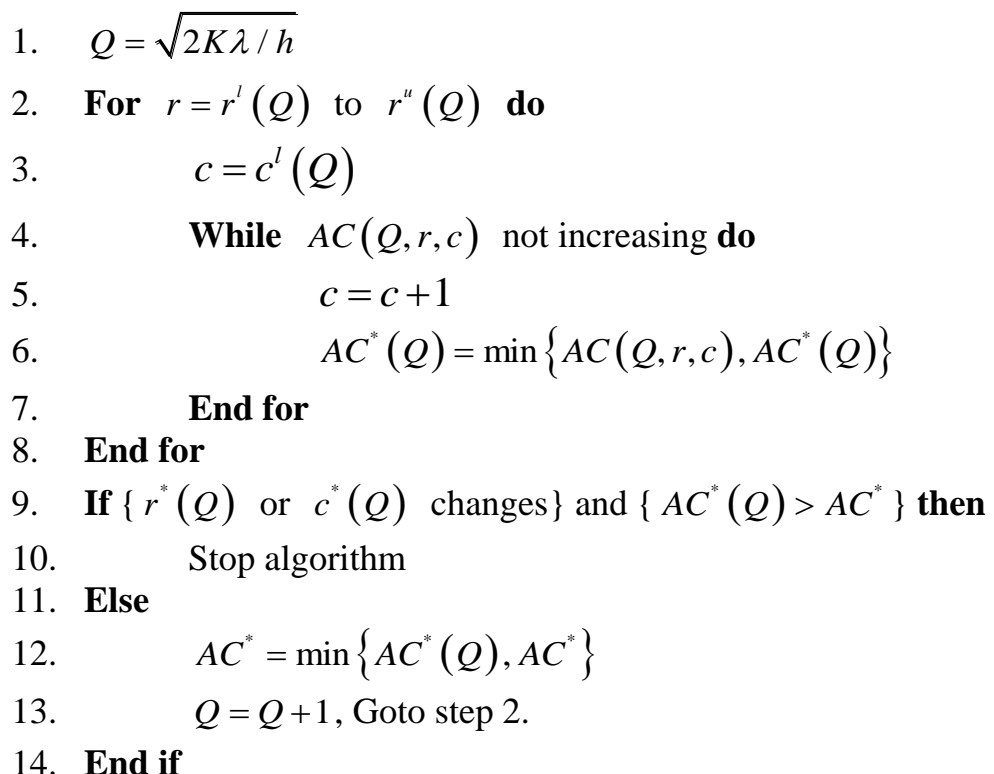

Again we have to note that our search method is different compared with the one in Deshpande et al. (2003), in which the authors fix $c$ and then search optimal $(Q, r)$ using the algorithm of Federgruen and Zheng (1992). It is fast in searching $(Q, r)$, but it may take a long time to obtain the optimal $c$ in an open space. However, in our heuristic, we propose 
the bounds and stop rules for all the decision variables thus the search range becomes more restrictive. We will present the performance of our heuristic in the following numerical analysis.

\section{Numerical analysis}

In this section, we present the numerical analysis. As illustrated in Figure 5, we have several purposes in making comparisons. First, we examine the performance of the threshold heuristic by comparing it with the optimal solutions obtained through complete enumeration. Secondly, we compare the heuristic with a modified method of Deshpande et al. (2003) to show the necessity of developing this heuristic. Thirdly, we compare the threshold clearing mechanism (using the heuristic solutions) with the priority clearing mechanism (decision variables are obtained from simulation and the heuristic, respectively) in order to understand their impact on the system performance. Finally, we compare the models with and without rationing $(c=0)$ policies in order to identify the conditions in which rationing is important.

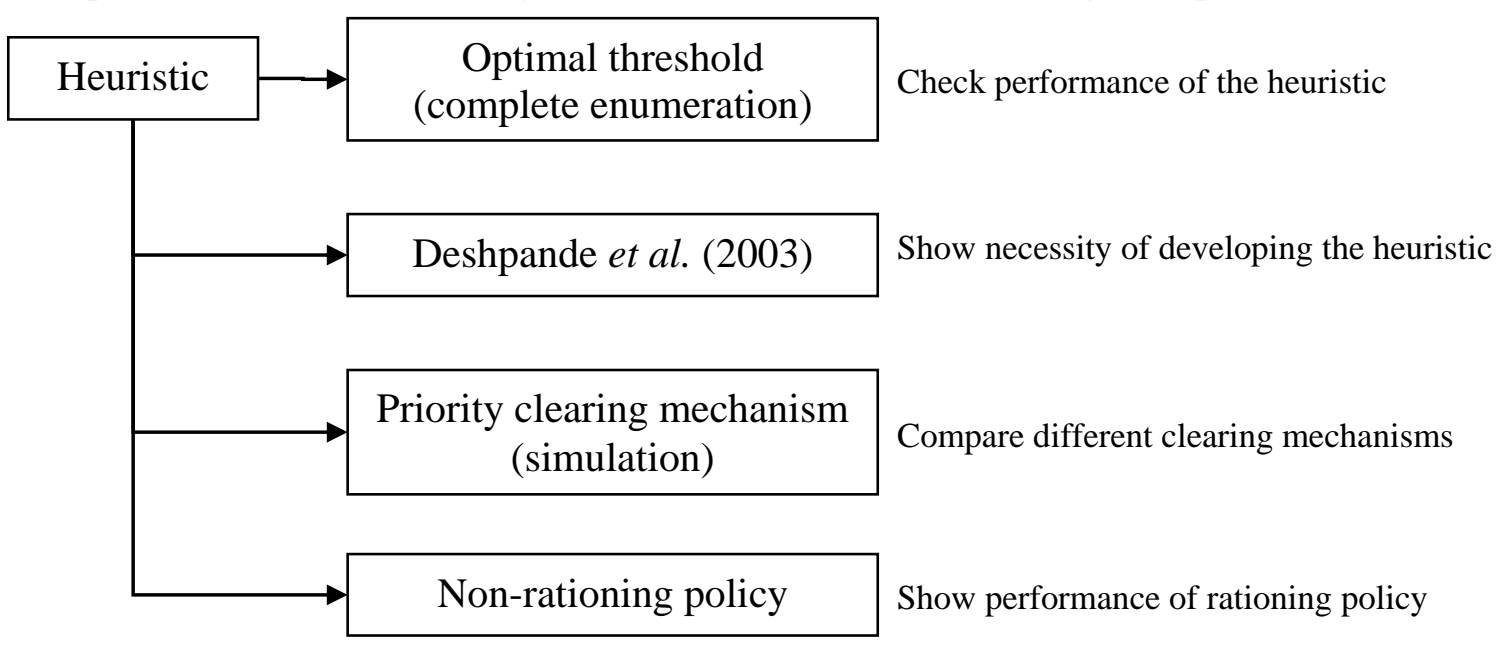

Figure 5. Structure of the numerical analysis.

To cover a wide range of data settings, we generated 756 problem sets varying in demand rate ratio $\left(\alpha_{1}=\lambda_{1} / \lambda=0.2,0.5,0.8\right.$, with $\left.\lambda=10\right)$, ordering cost $(K=0,20,50,100)$, lead time ( $L=1,3,5)$, minimal service level of Class $1\left(w_{1}=0.6,0.7,0.8,0.85,0.9,0.95,0.99\right)$ and backorder cost for Class $2\left(\pi_{2}=4,9,99\right)$. We fix the holding cost $h=1$.

\subsection{Heuristic algorithm performance}

The performance of the heuristic algorithm is compared with the optimal solutions obtained from complete enumeration. In both cases, the threshold clearing mechanism is applied. We implement these two algorithms in Mathematica 8.0. The PC has a CPU rate 2.7G. In the complete enumeration method, we search the optimal solutions within the ranges $Q \in\{1,100\}, r \in\{0,100\}, c \in\{0,20\}$, which after the preliminary test, we consider sufficient to obtain the optimal solutions for all the problem sets.

We find that the heuristic provides exactly the same solutions as the complete enumeration in all 756 problem sets. This good result indicates that the principle of the heuristic can be justified for our specific problem structure, and it also enables us to apply our heuristic to 
investigate other aspects of rationing policy, such as clearing mechanism, possibility of applying modified Deshpande et al. (2003) approach in the current problem.

In terms of computation time, the heuristic approach also performs well. Obviously the search by the complete enumeration is time-consuming. According to our search ranges, we need search $100 \times 101 \times 21=212100$ combinations for finding the optimal $(Q, r, c)$ for each problem set. On the other hand, in all 756 problems sets, due to the restrictive search ranges in our heuristic, each problem set tests an average 364.2 combination of $(Q, r, c)$ before the result is obtained. This accounts $0.17 \%$ compared with the case of complete enumeration. In fact, the heuristic generates the "near-optimal" (the same as optimal in our problems sets) solution within one second, whereas the complete enumeration takes about ten minutes.

Furthermore, in all the examples, the optimal $Q$ varies from 1 to 68. This range is almost five times wider compared with the examples considered in Deshpande et al. (2003). It indicates that our heuristic is also capable and competent with a large $Q$. In addition, it demonstrates that the search range $\{1, \ldots, 100\}$ for $Q$ in the complete enumeration is sufficient for ensuring the optimal solution. The above mentioned computation time make the heuristic possible to be implemented in practice.

\subsection{Comparison between heuristic and Deshpande's method}

As indicated in Section 3.3, one straightforward method to solve the mixed service criteria model is that we can transform $w_{1}$ to a penalty cost $\pi_{1}=h w_{1} /\left(1-w_{1}\right)$ and use the method in Deshpande et al. (2003) to obtain solutions denoted as $(Q, r, c)_{D}$. We then implement $(Q, r, c)_{D}$ in our mixed model and get the corresponding $A C_{D}$ and $F_{1, D}$, which are further compared respectively with the average cost $A C_{H}$ from the heuristic and the target service level $w_{1}$.

We have compared all 756 problem sets. Here we present the results of problems with $\pi_{2}=9$ in Table 1. Each cell contains two values indicated as the cost gap between Deshpande's method and our heuristic, and the service difference between Deshpande's method and the constraint. We notice that Deshpande's method cannot always guarantee a feasible solution for the mixed model. Compared with the heuristic, it provides either lower cost without satisfying the service constraint, or higher cost with unnecessary higher service level. In the heuristic, service is considered as a hard constraint, therefore it is always fulfilled. Whereas, using the modified cost approach as in Deshpande et al. (2003), this is not always the case. Especially when the service constraint is relatively high $\left(w_{1}>\pi_{2} /\left(h+\pi_{2}\right)=90 \%\right)$, Deshpande's method always provides lower service levels than the service constraints. In the worst case, it is $5.8 \%$ lower than the target value. In addition, its cost can unnecessarily increase up to $10.4 \%$. This result shows the necessity to develop a dedicated algorithm for the mixed service criteria model.

Another alternative to solve the unfeasible solutions generated by Deshpande's method is to change $\pi_{1}$. We can iteratively search $\pi_{1}$ until Deshpande's optimal solutions satisfy the service constraint. We first set the lower bound of $\pi_{1}$ as zero and the upper bound as a large 
value. Then we start the search from $\pi_{1}=h w_{1} /\left(1-w_{1}\right)$. If the Class 1 service level of Deshpande's optimal solutions $\left(F_{1, D}\right)$ is higher than $w_{1}$, we use this $\pi_{1}$ to replace the upper bound. Otherwise, we replace the lower bound. Then we use the average value of the lower bound and the upper bound as the new $\pi_{1}$ and calculate the optimal solution again. The iteration stops when the change of $\pi_{1}$ is less than $10^{-6}$. Although we can obtain feasible solutions in all the sets, the results are still unsatisfactory. First, the calculation becomes much more time-consuming. Second, Deshpande's method still generates high service with high costs in some problem sets. The difference between cost structures prevents Deshpande's cost-based model to obtain the optimal solutions in the mixed criteria model. Based on the analysis above, we believe our heuristic is valuable in both solution quality and calculation speed.

Table 1. A comparison of heuristic and Deshpande's method

$$
\left[\triangle A C \%=\left(A C_{D}-A C_{H}\right) / A C_{H}, \text { Service gap } \%=F_{1, D}-w_{1}\right]
$$

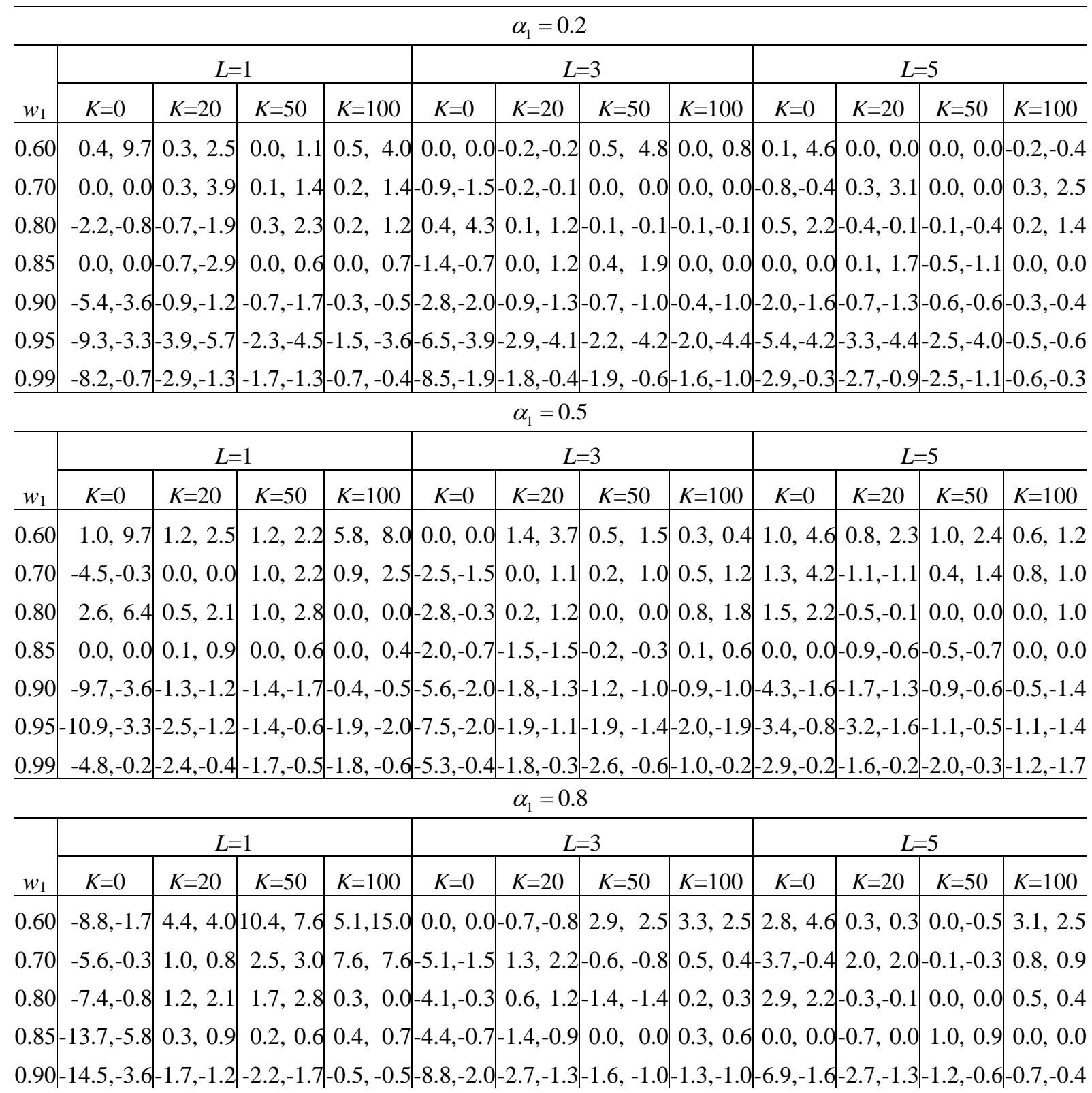


$0.95|-11.5,-2.6|-4.1,-2.2|-2.6,-1.8|-1.4,-1.0|-5.6,-1.0|-0.5,-0.2-1.4,-0.6|-1.9,-1.2|-6.1,-1.4|-3.1,-1.0|-1.8,-0.7 \mid-1.7,-0.9$ 0.99-11.8,-0.9-3.5,-0.6 -0.4,-0.1|-0.6, -0.1|-6.1,-0.5|-1.9,-0.2-0.8, -0.1|-1.9,-0.4|-2.4,-0.1|-3.2,-0.4-1.2,-0.2|-0.6,-0.1

\subsection{An evaluation of the clearing mechanism}

It is stated in Deshpande et al. (2003) that the priority clearing mechanism is optimal whereas the threshold mechanism is its approximation (for the sake of obtaining the analytical results). They compare two mechanisms and indicate that the threshold mechanism performs nearly the same as the priority one when ordering cost is not too small. Since our problem structure has changed, it is necessary to verify the performance of these two clearing mechanism. Based on the two clearing mechanisms, we study three clearing policies: the threshold clearing policy, the priority clearing policy and the hybrid clearing policy. When implementing the threshold clearing policy, we refer to a threshold clearing mechanism with decision variables solved from our heuristic (in previous section, the heuristic is shown to be accurate). Also we use complete enumeration with simulation approach in order to find the optimal solution for the priority clearing policy. We develop a $\mathrm{C}$ program to simulate 50 million demand events and search for the optimal solutions within the same intervals of $Q, r$ and $c$ as in Section 4.1. In addition, we include a hybrid policy, i.e. priority clearing mechanism is applied but the decision variables are obtained from our threshold heuristic. The last approach has the computation advantage in solving the decision variables.

We compare three policies for all 756 problem sets and find that in those sets with $K=20,50,100$, the threshold, hybrid and priority policies have the same results with regard to cost and service level. This is because a relatively large $K$ creates a large $Q$ which increases the chance to clear all backorders and bring the inventory level again above $c$. Thus there is no difference among the clearing mechanisms (recall the definition of the threshold mechanism in Section 3.2). We now reach a similar result as in Deshpande et al. (2003): with medium and high values of $K$, the threshold mechanism (with our heuristic) should provide a very good approximation for obtaining the optimal solutions.

Nevertheless, it is still interesting to investigate the cases with small $K$ values. Based on our examples, the situation has dramatically changed when $K=0$. Obvious performance differences exist among three policies, because $K=0$ leads to a small $Q$ and multiple outstanding orders. We present the comparison results of problem sets with $K=0, \pi_{2}=9$ in Table 2, which includes both cost and service level. Since the first part of clearing interval $\left(t_{j}, t_{c_{j}}\right)$ (cf. Figure 1) occurs more often, different clearing mechanisms in fact affect the performance measures.

Table 2. A comparison of threshold, hybrid and priority policies for $K=0$, [Cost gap $\%=\left(x-\right.$ priority)/priority, Service gap of $F_{1} \%=$ x-priority]

\begin{tabular}{|c|c|c|c|c|c|c|c|c|c|c|c|c|}
\hline \multicolumn{13}{|c|}{$\alpha_{1}=0.2$} \\
\hline \multirow[b]{2}{*}{$w_{1}$} & \multicolumn{4}{|c|}{$L=1$} & \multicolumn{4}{|c|}{$L=3$} & \multicolumn{4}{|c|}{$L=5$} \\
\hline & \multicolumn{2}{|c|}{$\begin{array}{l}\text { threshold } \\
\text { vs. } \\
\text { priority }\end{array}$} & \multicolumn{2}{|c|}{$\begin{array}{c}\text { hybrid } \\
\text { vs. } \\
\text { priority } \\
\end{array}$} & \multicolumn{2}{|c|}{$\begin{array}{c}\text { threshold } \\
\text { vs. } \\
\text { priority }\end{array}$} & \multicolumn{2}{|c|}{$\begin{array}{l}\text { hybrid } \\
\text { vs. } \\
\text { priority } \\
\end{array}$} & \multicolumn{2}{|c|}{$\begin{array}{c}\text { threshold } \\
\text { vs. } \\
\text { priority }\end{array}$} & \multicolumn{2}{|c|}{$\begin{array}{c}\text { hybrid } \\
\text { vs. } \\
\text { priority }\end{array}$} \\
\hline 0.60 & 9.2 & 5.7 & 2.9 & 5.7 & 21.0 & 0.1 & 4.9 & -0.1 & 27.4 & -0.1 & 6.7 & 0.0 \\
\hline 0.70 & 7.6 & 4.8 & 1.2 & 4.8 & 16.0 & 0.1 & 3.3 & 0.1 & 20.9 & 0.1 & 5.8 & 0.1 \\
\hline 0.80 & 4.9 & 0.0 & 0.0 & 0.0 & 10.4 & -0.1 & 0.0 & 0.0 & 14.8 & 0.1 & 1.1 & 0.0 \\
\hline
\end{tabular}




\begin{tabular}{|c|c|c|c|c|c|c|c|c|c|c|c|c|}
\hline 0.85 & 4.8 & 0.0 & 0.0 & 0.0 & 8.0 & 0.0 & 0.0 & 0.0 & 11.6 & 0.0 & 0.9 & -0.1 \\
\hline 0.90 & 0.0 & 0.0 & 0.0 & 0.0 & 0.0 & 0.0 & 0.0 & 0.0 & 0.0 & 0.0 & 0.0 & 0.0 \\
\hline 0.95 & -0.7 & 0.0 & 0.0 & 0.0 & -3.5 & -2.2 & 0.1 & 0.1 & -3.5 & -2.0 & 0.0 & 0.9 \\
\hline 0.99 & 7.5 & 0.1 & 8.1 & 0.4 & 3.1 & 0.0 & 5.4 & 0.7 & 1.5 & -0.2 & 3.3 & 0.7 \\
\hline \multicolumn{13}{|c|}{$\alpha_{1}=0.5$} \\
\hline \multirow[b]{2}{*}{$w_{1}$} & \multicolumn{4}{|c|}{$L=1$} & \multicolumn{4}{|c|}{$L=3$} & \multicolumn{4}{|c|}{$L=5$} \\
\hline & \multicolumn{2}{|c|}{$\begin{array}{l}\text { threshold } \\
\text { vs. } \\
\text { priority }\end{array}$} & \multicolumn{2}{|c|}{$\begin{array}{l}\text { hybrid } \\
\text { vs. } \\
\text { priority }\end{array}$} & \multicolumn{2}{|c|}{$\begin{array}{l}\text { threshold } \\
\text { vs. } \\
\text { priority }\end{array}$} & \multicolumn{2}{|c|}{$\begin{array}{l}\text { hybrid } \\
\text { vs. } \\
\text { priority }\end{array}$} & \multicolumn{2}{|c|}{$\begin{array}{c}\text { threshold } \\
\text { vs. } \\
\text { priority }\end{array}$} & \multicolumn{2}{|c|}{$\begin{array}{c}\text { hybrid } \\
\text { vs. } \\
\text { priority }\end{array}$} \\
\hline 0.60 & 17.3 & 0.0 & 0.0 & 0.0 & 36.8 & -0.1 & 21.9 & -0.1 & 39.9 & 0.2 & 13.6 & 0.3 \\
\hline 0.70 & 9.6 & -4.8 & 1.1 & -4.8 & 22.7 & -2.9 & 7.7 & -3.0 & 27.7 & -0.1 & 5.5 & -0.1 \\
\hline 0.80 & 8.0 & 0.0 & 0.0 & 0.0 & 14.0 & -2.4 & 3.6 & -2.4 & 18.0 & 0.0 & 1.3 & 0.1 \\
\hline 0.85 & 6.6 & 0.0 & 0.0 & 0.0 & 9.9 & -2.0 & 0.0 & 0.0 & 14.4 & 0.0 & 0.0 & 0.0 \\
\hline 0.90 & 0.0 & 0.0 & 0.0 & 0.0 & 0.0 & 0.0 & 0.0 & 0.0 & 0.0 & 0.0 & 0.0 & 0.0 \\
\hline 0.95 & -0.7 & -0.4 & 2.4 & 0.5 & 0.3 & -0.3 & 8.1 & 2.1 & -0.6 & -0.1 & 7.7 & 2.8 \\
\hline 0.99 & 2.5 & -0.2 & 3.9 & 0.1 & 5.4 & -0.1 & 8.3 & 0.5 & 6.9 & 0.0 & 14.2 & 0.9 \\
\hline \multicolumn{13}{|c|}{$\alpha_{1}=0.8$} \\
\hline \multirow[b]{2}{*}{$w_{1}$} & \multicolumn{4}{|c|}{$L=1$} & \multicolumn{4}{|c|}{$L=3$} & \multicolumn{4}{|c|}{$L=5$} \\
\hline & \multicolumn{2}{|c|}{$\begin{array}{c}\text { threshold } \\
\text { vs. } \\
\text { priority } \\
\end{array}$} & \multicolumn{2}{|c|}{$\begin{array}{l}\text { hybrid } \\
\text { vs. } \\
\text { priority } \\
\end{array}$} & \multicolumn{2}{|c|}{$\begin{array}{c}\text { threshold } \\
\text { vs. } \\
\text { priority } \\
\end{array}$} & \multicolumn{2}{|c|}{$\begin{array}{c}\text { hybrid } \\
\text { vs. } \\
\text { priority } \\
\end{array}$} & \multicolumn{2}{|c|}{$\begin{array}{c}\text { threshold } \\
\text { vs. } \\
\text { priority } \\
\end{array}$} & \multicolumn{2}{|c|}{$\begin{array}{c}\text { hybrid } \\
\text { vs. } \\
\text { priority } \\
\end{array}$} \\
\hline 0.60 & 17.8 & 0.0 & 12.8 & 0.0 & 26.1 & -0.1 & 9.1 & -0.1 & 24.9 & 0.0 & 3.3 & 0.0 \\
\hline 0.70 & 9.5 & 0.0 & 0.0 & 0.0 & 16.4 & 0.1 & 8.6 & 0.1 & 17.2 & -0.1 & 6.5 & -0.1 \\
\hline 0.80 & 4.4 & -0.1 & 0.0 & 0.0 & 8.4 & 0.1 & 0.0 & 0.0 & 11.2 & 0.0 & 0.0 & 0.0 \\
\hline 0.85 & 3.8 & 0.0 & 0.0 & 0.0 & 4.9 & 0.0 & 0.0 & 0.0 & 7.3 & 0.1 & 0.0 & 0.0 \\
\hline 0.90 & 0.0 & 0.0 & 0.0 & 0.0 & 0.0 & 0.0 & 0.0 & 0.0 & 0.0 & 0.0 & 0.0 & 0.0 \\
\hline 0.95 & -1.1 & 0.0 & 0.0 & 0.0 & -1.5 & -0.1 & 3.4 & 1.0 & -1.9 & -0.4 & 4.0 & 1.0 \\
\hline 0.99 & 2.4 & 0.0 & 5.8 & 0.3 & 4.0 & -0.1 & 7.6 & 0.4 & 4.3 & 0.0 & 13.3 & 0.7 \\
\hline
\end{tabular}

In order to compare three policies, we further plot the Pareto frontier diagrams in Figure 6, in which two examples with $\alpha_{1}=0.5, L=3, K=0$ and different $\pi_{2}$ values are presented. Be aware that the vertical axis indicates increasing of the service level of Class 1 , whereas the horizontal axis decreasing of the average cost. The clearing mechanisms have different performances largely depending on the definition of priority, i.e. the values of $w_{1}$ and $\pi_{2} /\left(h+\pi_{2}\right)$. In Figure 6, the solid symbols indicate the performance with $w_{1}<\pi_{2} /\left(h+\pi_{2}\right)$, while the hollow ones represent the case of $w_{1}>\pi_{2} /\left(h+\pi_{2}\right)$.

In the region of $w_{1}>\pi_{2} /\left(h+\pi_{2}\right)$, Class 1 has high priority. As we increase $w_{1}$ starting at the level $\pi_{2} /\left(h+\pi_{2}\right)$, the threshold clearing policy first performs better and then it is outperformed by the priority clearing policy. In Figure 6, we see a gradual shift of the outcomes from the two clearing mechanisms. This phenomenon exists also in the case of $\pi_{2}=99$, but due to the small range of $w_{1}>\pi_{2} /\left(h+\pi_{2}\right)$, it becomes less virtually recognised. When $w_{1}<\pi_{2} /\left(h+\pi_{2}\right)$, Class 2 has high priority and it also has its penalty cost in the 
objective function. The priority clearing policy dominates the threshold clearing policy. As indicated in Figure 6, the priority clearing policy always yields less cost while it still fulfils the service constraint.

The above observations are quite different from the ones in Deshpande et al. (2003), in which the authors argue that the priority clearing policy always dominates the threshold clearing policy. This difference is due to the different objective functions in the two studies. In Deshpande et al. (2003), penalty cost is the only criterion and it is charged at both demand classes, whereas in our study we have penalty cost only for one class (Class 2 in this case). Recall that during $\left(t_{j}, t_{c_{j}}\right)$, the priority mechanism clears more high priority backorders and less low priority ones, compared with the threshold mechanism. When $w_{1}$ is approaching $\pi_{2} /\left(h+\pi_{2}\right)$, there is a large shift of cost for priority clearing policy (see Figure 6). Even if the gap between $w_{1}$ and $\pi_{2} /\left(h+\pi_{2}\right)$ is tiny, the priority mechanism gives definite priority to the class with higher target service level and this may not always improve the objective function (such as $w_{1}>\pi_{2} /\left(h+\pi_{2}\right)$ ). It implies that the priority clearing over-emphasizes the distinction of the two classes even if it is not necessary. In fact when the priority of two classes cannot be obviously distinguished, threshold clearing policy is more appropriate, given the hard service constraint is fulfilled (in the case of $w_{1}>\pi_{2} /\left(h+\pi_{2}\right)$ ).

In both regions of $w_{1}>\pi_{2} /\left(h+\pi_{2}\right)$ and $w_{1}<\pi_{2} /\left(h+\pi_{2}\right)$, the hybrid clearing policy has a fairly close result compared with priority clearing policy, except in the case that $w_{1}$ is much smaller than $\pi_{2} /\left(h+\pi_{2}\right)$ (see Figure 6). This result is the same as in Deshpande et al. (2003). Thus the hybrid clearing policy can be a good representative of the priority clearing policy.

As a result, we propose the following principle for selecting the clearing policy: i) the hybrid clearing policy if $w_{1}$ is sufficiently larger than $\pi_{2} /\left(h+\pi_{2}\right)$; ii) the threshold clearing policy if $w_{1}$ is marginally larger than $\pi_{2} /\left(h+\pi_{2}\right)$; iii) the hybrid clearing policy if $w_{1}<\pi_{2} /\left(h+\pi_{2}\right)$. Comparing the two figures in Figure 6, we see that $\pi_{2}$ value also has its impact. With a smaller $\pi_{2}$, we have a smaller region with $w_{1}<\pi_{2} /\left(h+\pi_{2}\right)$ where the hybrid clearing policy has a good performance. As $\pi_{2}$ increases, the gap between the hybrid and priority clearing policies is enlarged in the region $w_{1}<\pi_{2} /\left(h+\pi_{2}\right)$. There is still potential to develop new models to improve the solution quality. 

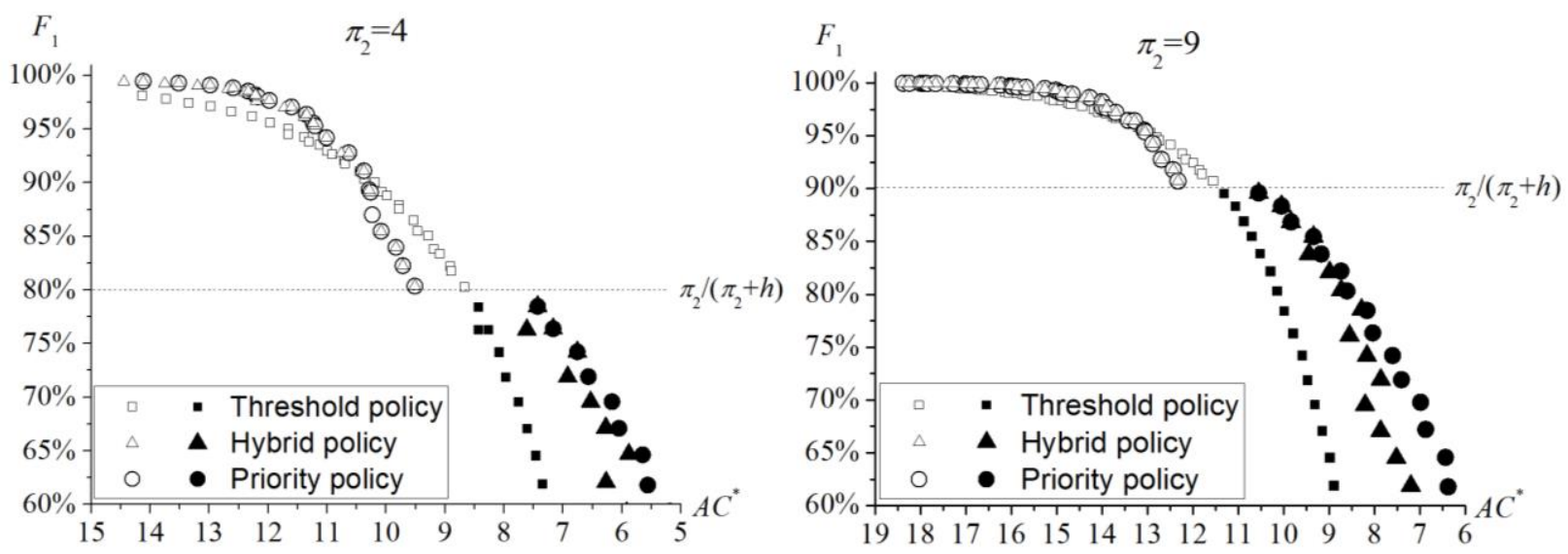

Figure 6. Pareto frontier of three policies.

\subsection{Rationing policy performance}

We now investigate the performance of rationing policy, i.e. in which circumstances rationing policy is needed and brings more benefits to the inventory system. Due to the good solution quality of our heuristic, we simply compare rationing policy (solution obtained from the heuristic) and non-rationing policy (optimal results obtained by complete enumeration with $c=0)$. Based on the comparison results, we notice that the rationing policy always outperforms the non-rationing policy in terms of cost. The cost gap is $5.3 \%$ on average and $42.2 \%$ as maximum. There are three scenarios in which the cost gaps are large and the rationing policy gains more benefits. First, when high priority demand has relatively small volume, the low priority demand dominates the total demand. Without rationing, inventory will be quickly consumed by the low priority demand, thus it creates unnecessary stockouts for high priority class. Secondly, when the difference between $w_{1}$ and $\pi_{2} /\left(h+\pi_{2}\right)$ increases, the rationing policy can distinguish the importance/requirement of the two classes effectively and therefore improve the performance. In the third case when the lead time $L$ is long, demand uncertainty will increase, thus the reorder point will rise in order to buffer the uncertainty. A rationing policy can provide target service levels and achieve the optimal balance among costs and service levels so as to reduce the unnecessary buffers for low class demand. To summarise, when we encounter these three scenarios, we should consider utilising rationing policy. 


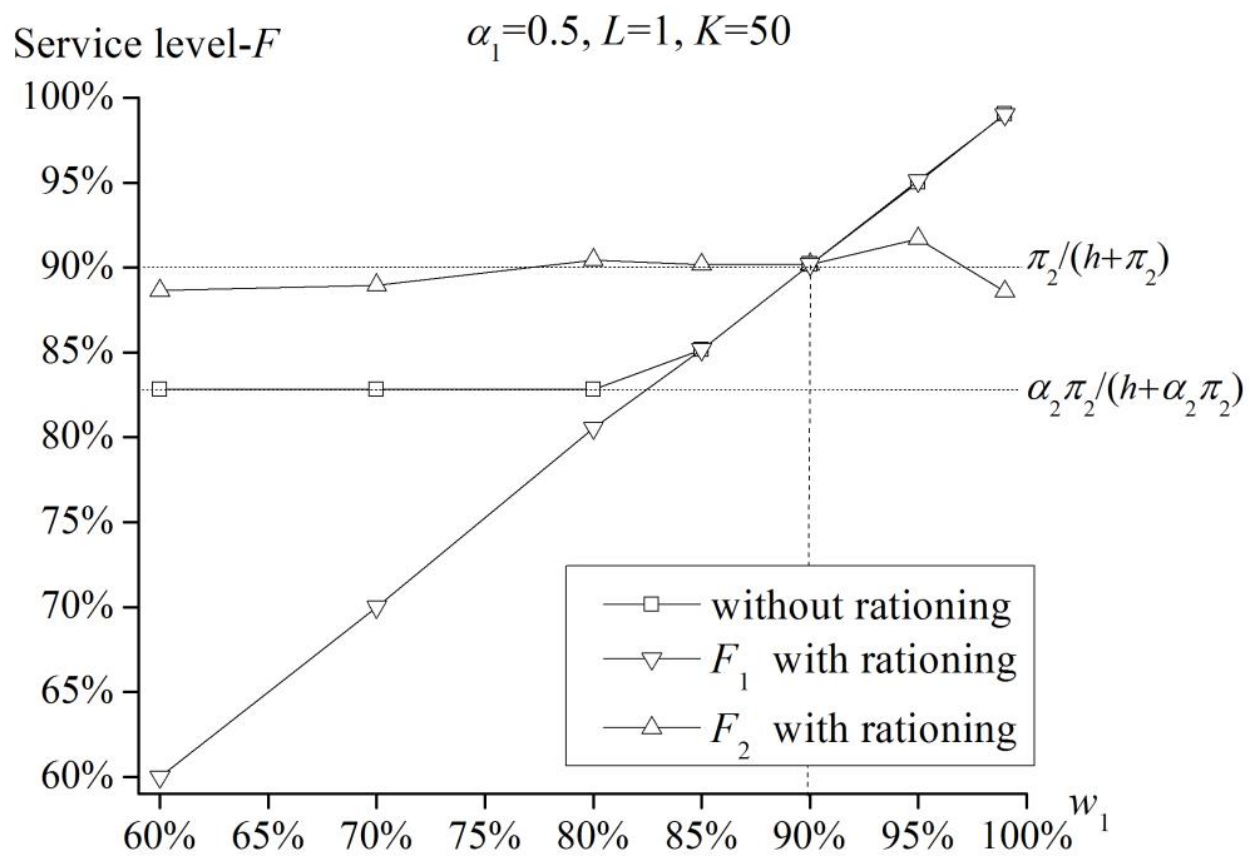

Figure 7. A comparison of service level between policies with and without rationing.

We also investigate the rationing effect in terms of service level. With $\alpha_{1}=\alpha_{2}=0.5, L=1$ and $K=50$ as an example, we illustrate here in Figure 7 the changes with and without rationing policies. We notice that the rationing policy always generates the service level close to the respective target values of each class. $F_{1}$ always stays with $w_{1}$ while $F_{2}$ fluctuates slightly around $\pi_{2} /\left(h+\pi_{2}\right)$ (due to the restriction of integral values). However, without rationing policy, there is always an aggregated service level $\max \left\{w_{1}, \alpha_{2} \pi_{2} /\left(h+\alpha_{2} \pi_{2}\right)\right\}$, which will overshoot/undershoot the target service levels of the respective classes.

According to the analysis above, we realise that the rationing policy performs better in terms of cost and service. Even though its modelling complexity increases substantially, its improvement of economic consequence is also obvious.

\section{Conclusion}

In this paper, we study an inventory model with two demand classes and mixed service criteria, i.e. penalty cost and service constraint. There are three major difficulties in dealing with such a problem: i) the priority of the demand class is not well defined due to the different dimensions of the performance criteria; ii) analytical expressions are not available for objective function and constraint when multiple outstanding orders exist and general clearing mechanism is allowed; iii) time-consuming search method is often needed for obtaining the solution in an open space.

We therefore propose some solutions for the above obstacles. First we transform the penalty cost to a target service level so as to define the priorities of demand classes. In order to develop the closed-form expressions to formulate this new problem, we further adopt the threshold clearing mechanism in Deshpande et al. (2003). Moreover, we develop the bounds and stop rules in the search method so as to make the proposed heuristic becomes 
computationally efficient.

Our numerical examples indicate that the heuristic performs very well. When a threshold clearing mechanism is used, our heuristic always generates the same result as the optimal one. Moreover, in our mixed performance criteria model, it is not a good approach to transform the service level constraint into a cost parameter and then apply solution method such as the one in Deshpande et al. (2003). In this case, cost will increase substantially or service level constraint will be violated.

Further investigation indicates that the clearing mechanism is important only when the setup cost is small (in our case $K=0$ ). We test the priority, hybrid and threshold clearing mechanisms and propose the conditions to implement them. Our study results are different from the one in Deshpande et al. (2003), in which the authors demonstrate that the priority clearing always outperforms the threshold clearing. However, this is not always true in our model due to the asymmetric service criteria of two classes. When the demand class with service constraint is has a marginally high priority, the priority clearing tends to quickly clear backlogs from the (marginally) high demand class whereas the clearance for the low demand class is pending. This in fact increases drastically the penalty cost in the objective function. The priority clearing is no longer an optimal clearing mechanism in our problem setting. Further development and investigation of the optimal clearing mechanism will be of great interest but also a challenge task in the future study.

Even though we attempt to investigate the rationing policy with different service criteria in this paper, we have limited our attention to a mixture of penalty cost and service level. Other performance measures may be further considered, such as lost sales, delivery lead time constraint and capacity utilisation. Moreover, it is also interesting to investigate the case that demand classes have different demand distributions with different values of mean and variance, in order to reflect various situations in industry.

\section{Acknowledgements}

We would like to express our great gratitude to the two anonymous referees. Their comments have helped significantly in revising and improving the quality of this manuscript. This research is partially supported by NSF of China under Grant 70832005 and partially performed within the Process Industry Centre (PIC) supported by the Swedish Foundation for Strategic Research (SSF).

\section{References}

[1] Agrawal, V., Seshadri, S., 2000. Distribution free bounds for service constrained $(Q, r)$ inventory systems. Naval Research Logistics, 47, 635-656.

[2] Axsäter, S., Kleijn, M., de Kok, T.G., 2004. Stock Rationing in a continuous review two-echelon inventory model. Annals of Operations Research, 126, 117-194.

[3] Axsäter, S., 2006. Inventory control. New Yorker: Springer Science.

[4] Chew, E.P., Lee, L.H., Teng, S., Koh, C.H., 2009. Differentiated service inventory optimization using nested partitions and MOCBA. Computers and Operations Research, 36(5), 1703-1710.

[5] Cohen, M.A., Kleindorfer, P.R., Lee, H.L., 1988. Service constrained $(s, S)$ inventory systems with priority demand classes and lost sales. Management Science, 34(4), 
482-499.

[6] Dekker, R., Kleijn, M.J., de Rooij, P.J., 1998. A spare parts stocking policy based on equipment criticality. International Journal of Production Economics, 56-57(20), 69-77.

[7] Deshpande, V., Cohen, M.A., Donohue, K., 2003. A threshold inventory rationing policy for service-differentiated demand classes. Management Science, 49(6), 683-703.

[8] Duran, S., Liu, T., Simchi-Levi, D., Swann, J.L., 2008. Policies utilizing tactical inventory for service-differentiated customers. Operations Research Letter, 36, 259-264.

[9] Fadiloglu, M.M., Bulut O., 2010. A dynamic rationing policy for continuous-review inventory systems. European Journal of Operational Research, 202(3), 675-685.

[10]Federgruen, A., Zheng, Y.-S., 1992. An efficient algorithm for computing an optimal $(r, Q)$ policy in continuous review stochastic inventory systems. Operations research, 40(4), 808-813.

[11] Kleijn, M.J., Dekker, R., 1998. An overview of inventory systems with several demand classes. Econometric Institute Report EI 9838, Erasmus University Rotterdam, Econometric Institute.

[12] Melchiors, P., Dekker, R., Kleijn, M.J., 2000. Inventory rationing in an $(s, Q)$ inventory model with lost sales and two demand classes. Journal of the Operational Research Society, 51(1), 111-122.

[13] Melchiors, P., 2003. Restricted time-remembering policies for the inventory rationing problem. International Journal of Production Economics, 81-82(11), 461-468.

[14] Moon, I., Kang, K., 1998. Rationing policies for some inventory systems. Journal of the Operational Research Society, 49, 509-518.

[15] Nahmias, S., Demmy, W.S., 1981. Operating characteristics of an inventory system with rationing. Management Science, 27, 1236-1245.

[16]Paul, B., Rajendran, C., 2011. Rationing mechanisms and inventory control-policy parameters for a divergent supply chain operating with lost sales and costs of review. Computers and Operations Research, 38(8), 1117-1130

[17]Platt, D.E., Robinson L.W. and Freund R.B., 1997. Tractable $(Q, R)$ heuristic models for constrained service levels. Management Science, 43, 951-965.

[18] Teunter, R.H., Haneveld, W.K.K., 2008. Dynamic inventory rationing strategies for inventory systems with two demand classes, Poisson demand and backordering. European Journal of Operational Research, 190(1), 156-178.

[19] Topkis, D.M., 1968. Optimal ordering and rationing policies in a nonstationary dynamic inventory model with $\mathrm{n}$ demand classes. Management Science, 15(3), 160-176.

[20] Veinott, A.F., 1965. Optimal policy in a dynamic, single product, nonstationary inventory model with several demand classes. Operations Research, 13, 761-778.

[21]Zheng, Y., 1992. On properties of stochastic inventory systems. Management Science, 38, 87-103.

[22]Zipkin, P., 2000. Foundations of inventory management. New York: McGraw-Hill. 